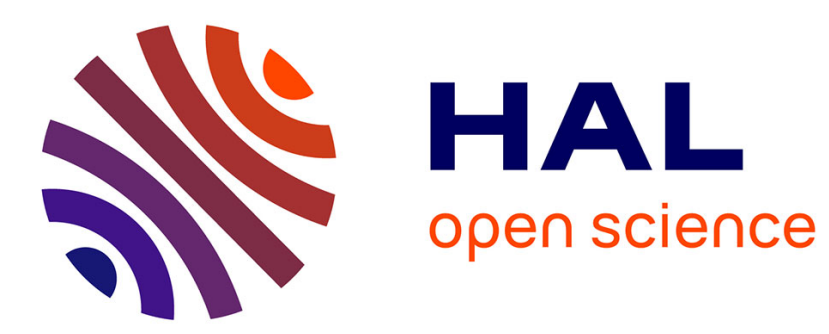

\title{
La société civile organisée et l'impératif participatif. Ambivalences et concurrence
}

Sandrine Rui

\section{To cite this version:}

Sandrine Rui. La société civile organisée et l'impératif participatif. Ambivalences et concurrence. Histoire, économie et société, 2016, Les corps intermédiaires en France: concept(s), généalogie et échelles, 35 (1), pp.58-74. 10.3917/hes.161.0058 . halshs-01348895

\section{HAL Id: halshs-01348895 https://shs.hal.science/halshs-01348895}

Submitted on 5 Sep 2016

HAL is a multi-disciplinary open access archive for the deposit and dissemination of scientific research documents, whether they are published or not. The documents may come from teaching and research institutions in France or abroad, or from public or private research centers.
L'archive ouverte pluridisciplinaire HAL, est destinée au dépôt et à la diffusion de documents scientifiques de niveau recherche, publiés ou non, émanant des établissements d'enseignement et de recherche français ou étrangers, des laboratoires publics ou privés. 


\title{
La société civile organisée et l'impératif participatif. Ambivalences et concurrence
}

\author{
Sandrine Rui
}

\section{Résumé}

Alors que, historiquement, leur rôle dans le modèle politique français a été progressivement réévalué, les acteurs de la société civile organisée affrontent avec scepticisme les développements contemporains de la démocratie participative. Mis en concurrence dans leur propre capacité autonome à investir et structurer l'espace public, ils doivent aussi compter avec un régime normatif contraignant qui altère leur capacité d'action et leur légitimité. Pourtant, la contribution des corps intermédiaires aux dispositifs participatifs s'avère non seulement décisive pour la qualité délibérative, mais elle est aussi une source de régénération dans un contexte où ils n'échappent pas eux-mêmes à la défiance.

\section{Abstract}

While their role in the French political model has been progressivily re-evaluated, the actors of organized civil society face with skepticism the contemporary developments of participatory democracy. In competition in their own autonomous ability to invest and structure the public sphere, they must also reckon with a restricting normative regime which distorts their agency and their legitimacy. Yet the contribution of organized civil society actors to participatory processes appears not only decisive for the deliberative performance, but it is also a source of regeneration in a context where they do not escape themselves from mistrust.

Comment les acteurs de la société civile organisée envisagent-ils les développements contemporains de la démocratie participative ? Cette question s'inscrit dans l'histoire longue des rapports entre l'État et la société, comme dans celle des liens consubstantiels entre société civile, espace public et démocratie. Elle retient surtout celle que Pierre Rosanvallon a retracée en décrivant les étapes et les arguments d'une progressive réévaluation de la place des corps intermédiaires dans la démocratie française ${ }^{1}$. Après une séquence révolutionnaire qui promeut la démocratie contre les corps intermédiaires, une tendance heurtée mais non démentie au libéralisme social a progressivement installé et conforté les

1. Pierre Rosanvallon, Le Modèle politique français : la société civile contre le jacobinisme de 1789 à nos jours, Paris, Seuil, 2004. Voir bien sûr aussi du même auteur : Le Peuple introuvable : histoire de la représentation démocratique en France, Paris, Gallimard, 1998. 
organisations de la société civile dans le jeu démocratique, tout en n'effaçant pas pleinement le penchant à l'illibéralisme politique, soit à la concentration du pouvoir politique. Cette tension structurante de ce que l'auteur qualifie de « jacobinisme amendé » explique l'écart entre les faits et les représentations, écart symptomatique du cas français : des rapports toujours plus institutionnalisés et soutenus par le droit entre pouvoirs et société civile organisée, mais aussi d'anciennes préventions toujours susceptibles d'être réactivées contre les corps intermédiaires, doublées d'une persistante déploration du centralisme. Or ces dernières décennies, en France, comme dans bien des sociétés démocratiques, le cadre législatif et réglementaire a consacré une exigence démocratique qui, sans être nouvelle, étend le domaine et les modalités d'intervention de la société civile : la participation directe des citoyens aux affaires qui les concernent ${ }^{2}$. Cet impératif participatif, aux soubassements théoriques et politiques anciens et renouvelés avec le tournant délibératif des années $1980^{3}$, a certes une portée limitée. La démocratie représentative reste le modèle dominant et comme l'écrit Guillaume Gourgues, " aucune "démocratie participative" de quelque forme que ce soit ne semble exercer une influence significative sur le gouvernement des affaires collectives $»^{4}$. Il reste que cette exigence se traduit désormais au concret dans des dispositifs et des procédures qui viennent troubler les rapports entre pouvoirs et corps intermédiaires. C'est ce trouble qu'il convient ici d'interroger.

Il est bien sûr délicat d'élaborer des raisonnements généraux à propos du "nouvel esprit de la démocratie $»^{5}$, tant il donne lieu à des dispositifs empiriques très divers. Ils renvoient à des procédures, des objets, des méthodes, des publics et des finalités hétéroclites, institués à différentes échelles territoriales : une conférence de citoyens n'est pas un conseil de quartier ; un débat public organisé par la Commission Nationale du Débat Public (CNDP) n'est pas un budget participatif, etc. Pourtant, par-delà leurs spécificités, ces dispositifs ont pour trait commun d'instituer des temps au cours desquels les citoyens sont invités à une mise en examen discursive, pluraliste et publique d'enjeux, de projets,

2. Parmi les textes, sans être exhaustif, on retiendra : la loi $n^{\circ} 83-630$ du 12 juillet 1983 relative à la démocratisation des enquêtes publiques et à la protection de l'environnement ; la loi nº 85-729 du 18 juillet 1985 relative à la mise en œuvre de principes d'aménagement (cf. l'article L 300-2 du Code de l'Urbanisme) ; la loi $\mathrm{n}^{\circ}$ 91-662 d'orientation sur la ville du 13 juillet 1991 ; la loi n $92-125$ du 6 février 1992 relative à l'administration territoriale de la République ; la loi n 95-101 du 2 février 1995, relative au renforcement de la protection de l'environnement ; la loi d'orientation $\mathrm{n}^{\circ} 99-533$ du 25 juin 1999 pour l'aménagement et le développement durable du territoire ; la loi n ${ }^{\circ}$ 2000-322 du 12 avril 2000 relative aux Droits des Citoyens dans leurs Relations avec les Administrations ; la loi ${ }^{\circ}$ 2000-1208 du 13 décembre 2000 relative à la solidarité et au renouvellement urbains ; la loi n 2002-276 du 27 février 2002, relative à la démocratie de proximité ; la loi n ${ }^{\circ} 2004-809$ du 13 août 2004 relative aux libertés et responsabilités locales; la loi n ${ }^{\circ} 2009-967$ du 3 août 2009 de programmation relative à la mise en œuvre du Grenelle de l'environnement et la loi n ${ }^{\circ} 2010-788$ du 12 juillet 2010 portant engagement national pour l'environnement ; la loi n ${ }^{\circ} 2012-1460$ du 27 décembre 2012 relative à la mise en œuvre du principe de participation du public, en application de l'article 7 de la Charte de l'environnement, procédant de la révision constitutionnelle de 2008 ; la loi n 2014-366 du 24 mars 2014 relative à l'accès au logement et à un urbanisme rénové.

3. L'œuvre de Jürgen Habermas y occupe une place centrale, en particulier par les controverses critiques qu'elle a suscitées et les perspectives théoriques ouvertes. Jürgen Habermas, L'Espace public [1962], Paris, Payot, 1993 ; id., Droit et Démocratie : entre faits et normes, [1992], Paris, Gallimard, 1997. Pour une mise en perspective historique des théories participationnistes et délibératives de la démocratie, qui constituent une abondante littérature, voir notamment : Crawford B. Macpherson, Principes et limites de la démocratie libérale [1971], Paris, La Découverte, 1985 ; John Elster, Deliberative Democracy, Cambridge, Cambridge University Press, 1998 ou encore John S. Dryzek, Deliberative Democracy and Beyond : Liberal, Critics, Contestations, Oxford, Oxford University Press, 2002.

4. Guillaume Gourgues, Les Politiques de démocratie participative, Grenoble, Presses Universitaires de Grenoble, 2013.

5. Loïc Blondiaux, Le Nouvel Esprit de la démocratie, Paris, Seuil, 2008. 
de mesures intéressant la collectivité. La singularité de ces dispositifs tient à cela : la sollicitation directe de citoyens ordinaires, soit des citoyens sans mandat ni statut dans l'ordre de la décision publique, et n'ayant d'ordinaire d'autres ressources pour peser sur les décisions que le vote, la mobilisation collective ou le recours en justice. Dans les textes, les dénominations d'acteur du territoire, d'habitant, d'usager, de société civile ou encore de public demeurent ainsi assez vastes pour que chaque citoyen puisse entrer en concertation. Plus encore, l'absence de droits politiques n'entrave en rien la participation : les mineurs ou encore les étrangers peuvent pénétrer des scènes participatives, qu'elles leur soient dédiées ou ouvertes à tous.

Le grand public n'est pourtant pas le seul à être sollicité. Les corps intermédiaires sont également la cible de l'appel à la participation. Ici encore, tout raisonnement général ne peut être que prudent à propos de la société civile organisée, tant la diversité des acteurs collectifs qui la composent, de leur degré d'organisation et d'institutionnalisation, de leur degré d'intégration et de capacité d'action, empêche d'y voir un monde homogène. Les rapports de pouvoir au sein même de la société civile ne doivent pas être occultés : rien de commun entre la force de frappe d'une organisation patronale nationale et celle d'une association locale de défense de l'environnement. Nous retiendrons surtout que les acteurs collectifs dont il s'agit ici existent toujours préalablement à l'offre publique de participation, qu'il s'agisse d'associations, d'organisations professionnelles, syndicales ou patronales ${ }^{6}$. Quel que soit leur degré d'institutionnalisation, ils ont en commun d'intégrer de façon plus ou moins forte une définition de leur identité, de leur adversaire et de l'enjeu de la lutte $^{7}$. Cette précision permet de distinguer notre réflexion des interrogations relatives aux effets des dispositifs participatifs en termes de structuration de la société civile : ces effets sont perceptibles quand, invités à débattre, des individus singuliers et isolés se mobilisent, s'organisent et se constituent en acteurs sociaux ${ }^{8}$. Représentants d'intérêts organisés et forts de leur rôle de médiation sociale, les corps intermédiaires savent par ailleurs pouvoir compter sur une place dans le régime néo-corporatiste de la décision, qui les intègre tant au fonctionnement de l'État qu'à la définition des politiques publiques. Ils comptent sur les sollicitations anciennes et continues de l'administration consultative ${ }^{9}$, mais aussi sur des instances représentatives pérennes (conseils économiques, sociaux et environnementaux régionaux par exemple), même si là encore, cette intégration n'est jamais pleinement ni également acquise.

En retenant avec Alain Chatriot et Claire Lemercier que le déploiement d'institutions intermédiaires « apparaît sans cesse pris dans une tension entre demande d'expertise de l'État pour connaître et gouverner la société et auto-organisation du social, dans ses différentes dimensions ${ }^{10}$, l'offre publique de participation réactualise cette tension selon des singularités que nous nous proposons d'explorer. Cette exploration s'appuie sur une

6. Nous n'envisageons pas ici la place des partis politiques, traitée par ailleurs. Voir Sandrine Rui, « Des partis politiques entre discrétion et discrédit. Réflexions sur le fait partisan dans les débats publics relatifs aux grands projets d'aménagement », dans Les Partis politiques à l'épreuve des procédures délibératives, dir. Antoine Roger et Rémi Lefebvre, Rennes, Presses universitaires de Rennes, 2009, p. 51-67.

7. Alain Touraine, La Voix et le Regard, Paris, Seuil, 1978.

8. Sandrine Rui, La Démocratie en débat : les citoyens face à l'action publique, Paris, Armand Colin, 2004.

9. Yves Weber, L'Administration consultative, Paris, L.G.D.J., 1968. Voir aussi : Michel Le Clainche, «L'administration consultative, élément constitutif ou substitut de la démocratie administrative ? », Revue française d'administration publique, 2011/1, p. 39-48.

10. Alain Chatriot et Claire Lemercier, «Les corps intermédiaires », dans Dictionnaire critique de la République, dir. Vincent Duclert et Christophe Prochasson, Paris, Flammarion, 2002, p. 691-698, ici p. 697. 
relecture de nos travaux empiriques portant tantôt sur des procédures participatives mises en œuvre dans le champ de l'aménagement, de la protection de l'environnement et des projets d'infrastructure, tantôt sur des dispositifs développés dans le cadre de la démocratie locale (conseils de quartier notamment) ou du renouvellement urbain et de la politique de la ville ${ }^{11}$.

Prompts à s'envisager comme les premiers défenseurs de la démocratie participative, les corps intermédiaires ne font cependant pas montre d'un grand enthousiasme à l'égard des dispositifs participatifs contemporains. Hostiles, défiants ou indifférents, leurs usages des espaces publics institutionnalisés sont de fait très ambivalents. Point de départ de notre réflexion, le scepticisme qui les anime n'est pas pour surprendre. Au début du XX siècle, lors des débats autour de la question syndicale, les promoteurs du syndicalisme défendent cette forme intermédiaire, fondée sur l'organisation économique de métier, sur l'organisation de la production et sur l'identité d'intérêts, contre ce qu'ils dénoncent comme un «démocratisme » prétendant impliquer les salariés selon des mécanismes de sollicitation directe ${ }^{12}$. Devenus des acteurs de la démocratie industrielle, les syndicats dénoncent encore régulièrement les appels à la participation directe des salariés dans l'entreprise, tant ils sont compris comme des stratégies pour les contourner et les affaiblir ${ }^{13}$.

S'il convient de rechercher les raisons des réticences dans les ambiguïtés de l'offre publique de participation, une attention doit aussi être portée à la concurrence qu' elle fait peser sur les corps intermédiaires. Par bien des aspects, ces derniers partagent les objectifs des dispositifs participatifs. Ils en subissent aussi les contraintes normatives. Pourtant, malgré ou en raison même des tensions, la participation des acteurs de la société civile organisée est une condition tant de la dynamique des scènes de débat public que de leur propre vitalité. Les développements contemporains de la démocratie participative constituent une ressource supplémentaire sur la voie du « jacobinisme amendé », qui, sans profondément renouveler les relations entre la puissance publique et les corps intermédiaires, offre une occasion de frottement et de dialogue avec les composantes singulières du corps social, source de régénération.

\section{Boycott, défiance, indifférence}

Les citoyens ordinaires conviés à débattre se montrent souvent sarcastiques à l'égard des dispositifs participatifs ${ }^{14}$. Mais c'est bien du côté des acteurs de la société civile organisée que la critique sociale est des plus consistantes ${ }^{15}$. Quels que soient les procédures, leurs instigateurs et leurs échelles, scepticisme et réticences caractérisent tout particulièrement les acteurs collectifs mobilisés et organisés, existant préalablement à l'offre publique de participation, qu'il s'agisse de corps intermédiaires institués de longue date ou de

11. Il y sera fait référence au fil du texte.

12. P. Rosanvallon, Le Modèle politique français, op. cit., p. 298 sq.

13. Voir par exemple: Dominique Martin, Démocratie industrielle : la participation directe dans les entreprises, Paris, Presses Universitaires de France, 1994 ; Julien Charles, Une Participation éprouvante: enquêtes sur l'autogestion, le management participatif, la participation citoyenne et l'empowerment, thèse de doctorat en sociologie, dir. Laurent Thévenot et Thomas Périlleux, EHESS/Université catholique de Louvain, 2012. Voir aussi le dossier Participation dans le monde du travail, dir. Sophie Béroud, numéro spécial de Participations, 2013/1, en particulier l'introduction, p. 5-32.

14. Rémi Barbier, « Quand le public prend ses distances avec la participation. Topiques de l'ironie ordinaire », Natures, Sciences, Sociétés, 2005/3, p. 258-265 ; Alice Mazeaud et Julien Talpin, « Participer pour quoi faire ? Esquisse d'une sociologie de l'engagement dans les budgets participatifs », Sociologie, 2010/3, p. 357-374.

15. Guillaume Gourgues, Sandrine Rui et Sezin Topçu, «Gouvernementalité et participation. Lectures critiques », Participations, 2013/2, p. 7-33. 
mouvements sociaux émergents. Ces attitudes rétives s'expriment selon des modalités diverses. Rares mais retentissants, les appels au boycott et au sabotage des débats publics s'observent tout particulièrement lors des procédures concernant la filière nucléaire ${ }^{16} \mathrm{ou}$ encore, ces dernières années, dans le domaine des nanotechnologies et des biotechnologies. Depuis le début des années 2000, le collectif Pièces et Main-d'œuvre revendique de n'appartenir à « aucun groupe - politique, syndical, associatif » et agit depuis son « site de bricolage pour la construction d'un esprit critique grenoblois ${ }^{17}$, tout en se rendant parfois sur les lieux des débats pour en contester la facture. Des organisations bien installées ont aussi eu recours au boycott, à l'instar de France Nature Environnement et de MirabelLNE, ou encore des composantes du réseau Sortir du nucléaire, comme ce fut notamment le cas, en 2013, lors du débat public relatif au projet Cigéo prévoyant l'enfouissement des déchets radioactifs à Bure dans la Meuse. Les acteurs collectifs engagés dans le réseau européen contre les Grands Projets Inutiles Imposés ${ }^{18}$ optent de la même manière pour la contestation des cadres procéduraux du dialogue et constituent en propre des espaces publics oppositionnels ${ }^{19}$ pour faire contrepoids aux « pseudo-concertations » qu'ils dénoncent.

On nous accuse d'avoir une position de principe où on refuse systématiquement de participer aux concertations mais ce n'est pas vrai. On constatait que tant pour les débats publics sur les réacteurs nucléaires, sur les déchets radioactifs, que pour le Grenelle, tout était plié avant. C'est se moquer du monde ! Donc, notre position était le boycott. On allait devant les étapes du débat public pour manifester, dénoncer cette parodie de démocratie. Cette ligne que certains vont percevoir comme radicale, moi, je la trouve cohérente. Je suis favorable à l'idée de concertation dans l'absolu mais dans les faits, ce sont des outils à disposition de ceux qui veulent imposer les projets. Le processus même de concertation est un instrument pour faire avaler la pilule. Nous, on n'a rien à attendre de la concertation. Pour nous, les débats publics, ils sont finalement utiles dans la mesure où c'est une bonne occasion d'aller devant pour manifester, pour dénoncer. Donc, on renverse : on instrumentalise le truc qui est prévu pour nous instrumentaliser. La concertation c'est une bonne opportunité pour contre-attaquer (ONG anti-nucléaire).

Les refus radicaux et proclamés de débattre demeurent toutefois exceptionnels. La défiance, elle, est bien générale. Les acteurs de la société civile organisée se méfient des tentatives d'instrumentalisation, tant ils entrevoient que les débats présentés comme devant asseoir la légitimité des décisions et des projets pourraient n'être que de pures entreprises de légitimation. Incriminant l'imperfection des dispositifs proposés, ils craignent les effets

16. Ce fut également le cas lors de la concertation Granite en 2000 ou encore lors du débat public relatif au projet EPR en 2006. Dans les deux cas, le réseau Sortir du nucléaire fut à l'origine de l'appel au boycott. Voir pour le premier cas : Sandrine Rui, Stéphane Chataignier, Sandrine Pierlot, « Le débat impensable ? L'enfouissement des déchets radioactifs au risque de la démocratie », dans La situation délibérative dans le débat public, dir. Bernard Castagna et al., Tours, Presses Universitaires François-Rabelais, 2004.

17. http://www.piecesetmaindoeuvre.com. Les prises de position de ce collectif sont sans ambiguïté : « Nous refusons les débats après fait accompli avec les scientifiques, les décideurs et les instances chargées des pseudoconcertations avec "le public". Nous combattons les sociologues spécialisés dans "l'étude des controverses" et l'acceptabilité sociale, c'est-à-dire dans la prévention et l'apprivoisement des contestations. Nous avons appris de leurs propres études que "faire participer, c'est faire accepter". D'où il suit que participer, c'est accepter. »

18. Le $4^{\mathrm{e}}$ forum social thématique européen contre les Grands Projets Inutiles Imposés (GPII) s'est tenu à Rosia Montana (Roumanie) en mai 2014, et le $5^{\mathrm{e}}$ à Bagnaria Arsa (Italie) en juillet 2015.

19. Oskar Negt, L'Espace public oppositionnel, Paris, Payot, 2007. 
de réduction et de dilution de leurs revendications, et surtout de leur force critique, par l'élargissement du dialogue à des participants moins aguerris qu'eux à la concertation avec les autorités publiques. Jugés moins représentatifs et moins compétents, ils sont perçus comme moins légitimes. Un représentant du monde agricole regrette ainsi que le développement de la participation s'accompagne d'un dévoiement des critères de représentativité :

Dans le Grenelle, nous les agriculteurs étions représentés. Mais notre sentiment c'est que dans la démocratie participative, que l'on soit l'association Perlimpinpin avec dix militants ou une organisation syndicale avec plusieurs centaines de milliers d'adhérents, il y a dans les deux cas une personne autour de la table. C'est n'importe quoi ! C'est un manque de respect énorme par rapport aux enjeux que nous représentons. C'est une offense. On y est allé car les absents ont toujours tort et que cela pouvait nous être reprochés.

L'hostilité à l'égard de l'extension du domaine de la participation tient aussi à l'idée qu'il est « démagogique » de faire « comme si les citoyens étaient tous des experts ». Aussi, comme le regrette ce même représentant d'une organisation agricole, mettre autour d'une table « des gens qui n'ont pas une connaissance suffisante pour traiter des sujets est ridicule. Se prononcer sur des choix techniques - pas d'OGM, pas de ci, pas de là, - par des gens qui n'y connaissent rien : non. C'est comme si moi j'allais réciter le Coran dans une mosquée ou traiter les malades en ville sans être toubib. On peut avoir des idées sur tout, mais on ne peut pas mettre les idées de tout le monde au même niveau que celles des professionnels. Il faut de l'expertise, de la compétence et de la représentativité ».

D'un terrain d'observation à l'autre, l'offre publique de participation apparaît à leurs yeux comme s'adressant principalement au grand public, et comme ne constituant pas une plus-value démocratique au regard de leurs propres logiques d'action et de communication, mais surtout de leur propre intégration aux circuits de dialogue de l'administration consultative d'une part, aux instances représentatives traditionnelles de la société civile organisée d'autre part. Le représentant d'une Union de pêcheurs l'assène : "L'exercice médiatique qu'a constitué le débat n'a pas d'intérêt. Ce qui compte c'est la façon dont on discute des enjeux en coulisse, avec partenaires et adversaires. » La condescendance, l'indifférence courtoise, ou encore l'adhésion distanciée des acteurs de la société civile sont de fait des attitudes fréquentes ${ }^{20}$. Aussi, s'ils pénètrent les scènes participatives, c'est sans renoncer aux répertoires d'action traditionnels qui permettent de peser sur les rapports de force qui les opposent tantôt à l'État et à sa "technostructure", tantôt aux autorités politiques territoriales, mais aussi aux autres composantes de la société civile organisée. De ce point de vue, le lobbying constitue une activité assurément plus décisive, activité tactique et officieuse qui s'accommode de l'ombre pour obtenir la prise en compte des intérêts et des causes défendus.

Une partie de mon activité consiste en du lobbying sans que cela ne soit officiel. Cela ressemble à de l'argumentaire autour d'un café en marge des réunions de concertation ; cela consiste en des coups de fil pour expliquer les problématiques et pour faire passer des dossiers ; cela consiste en des mails aux gens de l'administration,

20. Sandrine Rui, « Le public fait-il la loi ? Le débat national sur l'eau, entre injonction participative et néo-corporatisme », Politix, 2006/4, p. 125-142 ; Sandrine Rui et Agnès Villechaise, « Les associations face à la participation institutionnalisée. Les ressorts d'une adhésion distanciée », Espaces et Sociétés, 2005/4, p. 21-37. 
en disant : voilà un argumentaire pour faire passer tel dossier, telle idée ; vous avez besoin d'arguments scientifiques pour - je l'ai fait encore hier soir - appuyer un dossier, il se trouve que moi j'ai la bibliographie qu'il faut, donc je fais passer le document qui permet de faire pencher la balance. (Organisation professionnelle)

Nous avons des représentants à Bruxelles qui font un lobbying intense pour que la France modifie ses lois, pour qu'elle se mette en conformité avec la réglementation européenne. Il y a le bureau européen de l'environnement, c'est pour nous une des composantes de la modification des lois. Ensuite il y a le dernier recours, la Cour européenne de justice. Ce lobbying européen intense est indispensable. (Organisation environnementaliste)

Bien sûr l'asymétrie des rapports de force entre acteurs organisés de la société civile doit être ici convoquée. Pour les groupes d'intérêts socio-économiques, l'imperfection des textes et des dispositifs participatifs est perçue comme une garantie que le jeu des pressions et de l'influence ordinaire ne sera troublé ni par une publicité dommageable ni par l'élargissement aux citoyens. C'est l'attitude dominante à l'égard du mécanisme de consultation introduit par l'article 244 de la loi n ${ }^{\circ} 2010-788$ du 12 juillet 2010 portant engagement national pour l'environnement (dite Grenelle 2), qui prévoit la participation du public aux projets de décisions réglementaires de l'État et de ses établissements publics lorsqu'elles ont une incidence significative sur l'environnement ${ }^{21}$. Au mieux concèdent-ils, comme ce représentant d'une organisation patronale, que cette disposition pourrait permettre de formaliser les démarches habituelles et de donner plus de poids aux revendications :

C'est-à-dire qu'aujourd'hui on formulait un avis, on faisait remonter un courrier à en-tête en disant que les entreprises n'allaient pas être très contentes de telle loi. C'était une communication officielle mais qui n'avait pas plus de poids que ça. J'ai l'impression, mais encore faut-il voir dans les faits, qu'avec cette loi ça permettrait de donner tout de suite une légitimité et une écoute à la requête. La consultation du public permettra peut-être de donner un caractère plus officiel à des processus qui existent déjà de dialogue. Mais il y aura toujours des négociations entre initiés. Ce n'est pas pour cacher les choses, mais il y a des problématiques extrêmement complexes qui ne gagneraient rien à être débattues sur la place publique. Exemple : le modèle d'établissement des tarifs de l'électricité. Honnêtement il n'y a qu'en France qu'on est capable de faire ça avec une armée de polytechniciens.

Du côté des organisations environnementalistes ou des associations de consommateurs et d'usagers, on attend généralement une consolidation juridique de la démocratie participative. Un surcroît de concertation juridiquement encadrée promet de réduire l'asymétrie des rapports de force qui leur est défavorable. « J'ai été auditionné à ma demande par les services de l'État, sans difficulté », se souvient ce représentant écologiste, « le problème, c'est le lobbying, comme sur l'eau ou les autres enjeux environnementaux. De façon générale, on est toujours en position de faiblesse. Les associations environnementalistes sont faibles : c'est 15 papys, le poids est limité. C'est tout le problème du mouvement écologiste en général. » En matière de production réglementaire notamment, si l'exemple

21. Cécile Blatrix et al., «Soumettre au public l'activité réglementaire en matière d'environnement: exigence démocratique, nécessité juridique et difficultés pratiques », dans Environnement : la concertation apprivoisée, contestée, dépassée ?, dir. Laurent Mermet et Denis Salles, Bruxelles, De Boeck, 2015, p. 169-186. 
de l'Union Européenne est régulièrement salué, c'est sans doute car son modèle légitime la participation des groupes d'intérêt, tout en l'encadrant par des mécanismes de contrôle et des mécanismes de participation citoyenne ${ }^{22}$. La pratique lobbyiste tend à gagner en légitimité, mais personne n'oublie qu'elle participe aussi «d'une distorsion subtile du processus démocratique ${ }^{23}$. Il reste qu' offre publique de participation ou pas, le répertoire d'action traditionnel ne saurait être délaissé sous peine de perdre sur tous les fronts. Pour les corps intermédiaires, mobilisations collectives, actions au plus près du législateur et des décideurs, et recours en contentieux ont donc encore de l'avenir.

\section{L'institutionnalisation de la participation contre les corps intermédiaires ?}

Les attitudes des corps intermédiaires à l'égard de l'offre publique de participation tiennent pour une part aux ambiguïtés de cette dernière. Le développement contemporain de l'exigence participative et sa consolidation juridique doivent pour partie aux revendications directes de la société civile, et notamment des mouvements sociaux. Cécile Blatrix a ainsi montré comment, parallèlement à des mouvements d'origines administrative et politique, les mouvements de la société civile avaient travaillé depuis la fin des années 1960 à élargir les droits à la participation démocratique ${ }^{24}$. Les Nouveaux Mouvements Sociaux résistaient alors à toute forme d'aliénation politique et culturelle et luttaient pour le contrôle du changement, considérant que « la forme la plus grave de gaspillage (était) l'absence de participation à la décision $»^{25}$. De fait, les demandes d'information, de participation aux décisions et aux organisations ainsi que le pouvoir d'auto-détermination constituaient le cœur des luttes. Mais, si le mouvement ouvrier a fondé la démocratie industrielle, la diversité des mouvements de la société post-industrielle et leur absence d'unité expliquent sans doute que la démocratie "sociale" n'ait jamais été ni très consistante ni très autonome. Les acteurs des nouveaux mouvements sociaux ont surtout progressivement gagné une place dans une configuration politique néo-corporatiste. Selon une logique d'incorporation ${ }^{26}$, certaines composantes des mouvements sociaux se sont imposées comme des partenaires de l'action publique, devenant notamment des co-gestionnaires des ministères.

Cette intégration au système politique a conféré une capacité d'action importante aux acteurs des nouveaux mouvements sociaux. Elle a aussi affaibli leur capacité de représentation sociale. En grandissant, cet écart explique pour partie le regain de la conflictualité des années 1990 selon des luttes éparses aux logiques et aux registres d'action diversifiés. Dans bien des secteurs d'intervention publique, l'accroissement des crises, des conflits et des contentieux a eu tôt fait d'être perçu par le législateur comme le résultat de la faiblesse des corps intermédiaires qui peinaient à jouer leur rôle de médiation et de régulation sociale. Intégrés à la démocratie spécialisée, soit à un modèle qui croit à l'existence d'un centre politique ${ }^{27}$, les acteurs de la société civile organisée n'étaient pas en mesure de relayer la "nouvelle culture politique" exprimée par des mobilisations citoyennes

22. Sabine Saurugger, «Les groupes d'intérêt entre démocratie associative et mécanismes de contrôle », Raisons politiques, 2003/2, p. 151-169.

23. Edward B. Arroyo, «Les lobbies dans la démocratie », Projet, 2004/2, p. 60-65.

24. Cécile Blatrix, La Démocratie participative, de mai 1968 aux mobilisations anti-TGV : processus de consolidation d'institutions sociales émergentes, thèse de doctorat en science politique, dir. Daniel Gaxie, Université Paris 1, 2000.

25. Alain Touraine, La Société post-industrielle, Paris, Denoël, 1969, p. 88.

26. Marco Giugni, « Social Movements and Change : Incorporation, Transformation, and Democratization », dans From Contention to Democracy, dir. Marco Giugni, Douglas MacAdam et Charles Tilly, Lanham, Rowman \& Littlefield, 1998, p. XI-XXVI.

27. Ulrich Beck, Risk Society, Towards a New Modernity, London, Sage Publications, 1992. 
de toute facture, affranchies des fonctions de représentation et de négociation, concentrées sur celles de vigilance et de dénonciation ${ }^{28}$, et dessinant un espace de l'action collective de surcroît très fragmenté ${ }^{29}$.

L'institutionnalisation des dispositifs participatifs trouvait alors une double justification. De façon particulièrement vive dans les champs de l'aménagement et des infrastructures de transport, dans celui de la protection de l'environnement ou encore dans celui de la politique de la ville, elle est à la fois une réponse politique et procédurale à la faiblesse des corps intermédiaires et à la fois l'occasion de desserrer l'emprise de ces mêmes corps constitués devenus co-gestionnaires de l'action publique. L'appel à la participation directe du citoyen est donc motivé par ce diagnostic contraire : des corps intermédiaires à la fois trop forts et trop faibles. Aussi l'offre publique de participation s'avère-t-elle toujours ambivalente. D'une part, elle prétend démocratiser la démocratie : ses instigateurs entendent corriger les défaillances du système démocratique de délibération et de représentation, notamment en réactualisant et en élargissant le domaine d'application des principes normatifs de l'espace public libéral et en étendant la surface de la société civile impliquée. Mais, dans le même temps, elle entérine l'idée que la structuration de l'espace public ne peut être confiée aux seuls mouvements et acteurs de la société civile : ses cadres et ses normes sont pensés de façon à limiter tout autant leur charge subversive que leur emprise. L'institutionnalisation de la participation a tout lieu d'être perçue comme une menace pour les capacités d'action et de représentation des acteurs organisés de la société civile. Reste une ambiguiité jamais pleinement levée : à quelques dispositifs près, comme les conférences de consensus ou les jurys citoyens qui cherchent à constituer en « mini publics» des « panels citoyens » épurés de tout porteur d'intérêts, les autres dispositifs s'adressent à la fois à chaque citoyen sans distinction et à la fois aux acteurs des territoires et aux corps constitués, mêlant d'ailleurs acteurs de la société politique et acteurs de la société civile. Deux acceptions de la société civile se confondent donc généralement ${ }^{30}$ : l'une très lâche (tout le monde, la société) et l'autre renvoyant à sa dimension organisationnelle. De fait, un dispositif participatif organise une coprésence qui génère en continu des conflits de légitimités dans le processus de construction de l'intérêt général et de figuration de la volonté générale. Surtout, toute offre publique de participation vient concurrencer sur leur propre terrain les corps intermédiaires.

\section{Concurrence et ressource}

Tout acteur de la société civile organisée tire son assise démocratique de sa capacité à constituer en propre des « arènes publiques » selon des normes de communication qu'il maîtrise. Ainsi lorsque Daniel Cefaï retient que « les mobilisations collectives constituent des laboratoires d'action publique, des réseaux d'alerte et de vigilance, d'enquête et d'expérimentation, contribuent par leurs dénonciations et leurs revendications à la construction des problèmes publics, polarisent autour d'eux le déploiement de micro-arènes publiques dans lesquelles participent et interagissent de multiples protagonistes, de droit privé et de droit public »- étant dès lors « au cœur de la démocratie technique, participative ou délibérative ${ }^{31}-$, une telle définition s'ajuste tout autant aux formes émergentes de

28. Pierre Rosanvallon, La Contre-démocratie : la politique à l'âge de la défiance, Paris, Seuil, 2006.

29. François Dubet et Danilo Martuccelli, Dans quelle société vivons-nous ?, Paris, Seuil, 1998 ; Lilian Mathieu, L'Espace des mouvements sociaux, Paris, Le Croquant, 2012.

30. Jean Leca, « De la lumière sur la société civile », Critique internationale, 2003/4, p. 62-72.

31. Daniel Cefaï, Pourquoi se mobilise-t-on ?, Paris, La Découverte, 2007, p. 715. Dans cet ouvrage consacré aux théories des mobilisations collectives, l'auteur propose d'ailleurs un tableau synthétisant les différents paradigmes de l'action collective comprenant les formes de la démocratie participative (p. 22). 
la participation institutionnalisée qu'à l'action des corps intermédiaires. Sans compter que l'offre publique de participation s'ajoute à des scènes existantes d'interactions et de représentation institutionnelle de la société civile qui demeurent prisées, dès lors qu'elles paraissent toujours mieux régler la tension entre représentativité et efficacité. Mais cette même concurrence explique aussi que les corps intermédiaires, en dépit de leurs réticences, ne perdent pas de vue la ressource que constitue l'offre publique de participation.

La concurrence concerne d'une part le travail de construction et d'exploration des problèmes publics, essentiel pour éclairer la décision. Tout dispositif participatif prétend ouvrir un cadre pluraliste pour le traitement des divergences d'intérêt, selon la double dimension au cœur des tensions et conflits sociaux contemporains rappelée par Hervé Pourtois $^{32}$ : d'une part, la question de l'identification des faits et des conséquences; d'autre part, celle des significations sociales des pratiques. Le premier point s'illustre par la prégnance des questions de connaissance et d'évaluation, particulièrement vives dans une société du risque en proie aux incertitudes et aux doutes. Quels que soient les causes et les intérêts défendus, les acteurs de la société civile organisée fondent leurs actions et leurs organisations sur des savoirs et des expertises qui leur permettent d'éclairer (mais aussi de contredire) les autorités politiques et administratives. En situation de débat public, ces capacités d'expertises sont mises en concurrence pour l'établissement des faits et leur évaluation. L'évaluation pluraliste et controversée des projets et décisions, au regard des gains et des risques sociaux, économiques et environnementaux, structure pour une bonne part les échanges. Les formes participatives prévoient d'ailleurs parfois des moyens techniques ou financiers pour des compléments d'études et d'expertise, comme les débats publics initiés par la Commission Nationale du débat Public. L'objectif est bien de créer les conditions d'une information et d'une discussion critique des outils et des résultats de l'évaluation, que l'on se positionne ex ante ou ex post. Pour tous les participants, ces questions sont alors étroitement liées à un enjeu de reconnaissance du pluralisme des expertises $^{33}$.

D'autre part, les conflits sociaux contemporains tiennent au pluralisme des significations sociales des pratiques. Les expériences sociales singulières ou collectives donnent lieu à de multiples interprétations et conflits de définition. Quel sens assigner au rassemblement des jeunes dans les halls des habitats collectifs ? une pratique de loisirs et de sociabilité ? le lieu où se fomentent des incivilités ? Quel sens peuvent bien avoir les freins à la circulation automobile dans les centres urbains ? une mesure de protection de l'environnement ? une atteinte à l'autonomie des salariés résidant dans le périurbain ? Que promettent les nanotechnologies ? Une innovation salutaire pour les secteurs industriels et médicaux ? un risque pour les libertés individuelles ? une technologisation inquiétante de l'humain? Le sens du juste et de l'injuste, de l'acceptable et de l'inacceptable, est exploré au regard de la diversité des modèles culturels. Cette dimension culturelle et sociétale des conflits apparaît aussi lorsque s'affrontent des conceptions différentes du changement. Des opérations de renouvellement urbain aux projets d'infrastructure de transport, des objets divers sont l'occasion de réinterroger les choix de société et les théories du changement social qui les sous-tendent. L'enjeu ici est celui de la reconnaissance du pluralisme des significations et des orientations culturelles.

32. Hervé Pourtois, « Délibération, participation et sens du désaccord », Éthique publique, 2005/1, p. 145154.

33. La Société civile : savoirs, enjeux et acteurs en France et en Grande-Bretagne, 1780-1914, dir. Christophe Charle et Julien Vincent, Rennes, Presses universitaires de Rennes, 2011. 
Pour les acteurs de la société civile organisée, l'offre publique de participation est donc l'occasion d'une mise en concurrence du rôle qui est le leur, celui d'alerter, d'éclairer et de convaincre les décideurs ${ }^{34}$. L'enjeu est de parvenir à une certaine effectivité normative ${ }^{35}$ : il s'agit d'obtenir des autorités que les revendications se transforment en actions et en règles légitimes afin qu'elles puissent s'imposer à tous et dans la durée, pour orienter tant les comportements sociaux ordinaires que les pratiques des acteurs publics et privés.

L'offre de participation institutionnalisée concurrence également le travail des corps intermédiaires en matière de formation de l'opinion publique et de production de la volonté générale. Par l'information et la discussion, les dispositifs participatifs cherchent à construire un public, à l'éclairer et à l'enrôler dans la controverse. La sollicitation du grand public est défendue en ce qu'elle promet l'émergence d'arguments inédits, portés par des voix sociales bien "réelles", mais jusque-là inaudibles. Pour les corps intermédiaires, cette sollicitation directe des citoyens est à la fois perçue comme vaine et menaçante. Elle vient surtout concurrencer leurs propres objectifs de « régulation corrective » des distorsions caractérisant l'espace public «sauvage », du fait de l'inégale répartition du pouvoir social ${ }^{36}$. Les méthodologies participatives mises en œuvre comme les acteurs de la société civile organisée cherchent à compenser la faiblesse des individus atomisés en abaissant notamment le coût de leur participation. Or, dans les dispositifs participatifs, les corps intermédiaires se trouvent en coprésence d'un public qu'ils n'ont pas contribué à construire, et dont ils ne peuvent assurer la représentation. Ils sont tenus alors de justifier, comme tout participant, la légitimité de leur entrée en concertation. Toutefois, le public est également la cible des dispositifs délibératifs et des actions des corps intermédiaires qui cherchent aussi à l'informer, le former, voire modifier ses représentations et ses comportements. De fait, il s'agit toujours aussi d'instrumentaliser l'opinion publique afin de reconfigurer un système d'action que l'on veut maîtriser : quand les instigateurs d'espaces publics institutionnalisés prétendent "libérer" le public de l'entrave que constitue la prédominance des corps constitués, ces derniers entendent l'émanciper des pouvoirs centraux. De fait, si les citoyens ordinaires viennent semer le trouble dans les logiques d'action des corps intermédiaires, les scènes participatives deviennent aussi des ressources pour concerner, mobiliser voire organiser ces participants perçus comme désassociés, peu clairvoyants et attachés à leurs seuls intérêts singuliers.

\section{Un régime normatif contraignant}

Cette volonté partagée de compléter et de contrarier la démocratie de la légitimité électorale $^{37}$ trouve toutefois son point limite dans les normes de délibération, de représentation et d'action qui gouvernent généralement les dispositifs participatifs. Si elles ne s'imposent jamais pleinement au concret, ces normes entrent en tension avec les propres attendus et rationalités des acteurs de la société civile organisée qui, de fait, ont toutes les raisons d'être réticents et ambivalents.

Les espaces publics institutionnalisés sont initiés et structurés par des autorités puisant leur légitimité aux sources de la démocratie électorale, de façon directe ou indirecte. La qualité des instigateurs et la procédure précipitent une scène de dialogue adossée aux

34. Michel Callon, Pierre Lascoumes et Yannick Barthes, Agir dans un monde incertain, Paris, Seuil, 2001.

35. Guy Groux, « Crise de la médiation et mouvements sociaux : vers une démocratie de l'entre-deux ? », dans Le Désenchantement démocratique, dir. Pascal Perrineau, La Tour d'Aigues, Éditions de 1'Aube, 2003, p. 231-242.

36. J. Habermas, Droit et Démocratie, op. cit.

37. P. Rosanvallon, La Contre-démocratie, op. cit. 
instances démocratiques traditionnelles : le public qui en résulte est un public relativement fort, dont le coût est supporté par la collectivité et dont la légitimité découle du cadre procédural. Tout corps intermédiaire a de son côté une pratique autonome de constitution d'arènes publiques, au moyen de répertoires d'action divers pour donner visibilité, légitimité et consistance à ses revendications. Ils peuvent constituer des scènes discursives en propre (meeting et manifestation, magazines, site internet et forum virtuel...) mais aussi opter pour le forçage d'arènes traditionnelles multiples (politique, administrative, médiatique, judiciaire, scientifique...). Pour les corps intermédiaires, une telle activité discursive démultipliée et à géométrie variable est indispensable, qu'ils poursuivent des objectifs offensifs ou défensifs ${ }^{38}$.

Or les dispositifs participatifs et délibératifs se présentent comme des temps au cours desquels il convient de se concentrer sur les strictes exigences du dialogue et de l'argumentation, le cadre procédural constituant l'unité de lieu et de temps de l'échange. Tout type d'action doit alors être suspendu et différé. Ce principe est énoncé de façon plus ou moins explicite, et s'adresse à l'ensemble des participants. Qu'une mesure soit mise en œuvre par une collectivité alors même que les citoyens sont conviés à débattre de son bien-fondé, et les participants jugeront avoir été trompés. Rendre publique une décision alors même qu'un débat public n'est pas allé à son terme est de nature à entacher la procédure ${ }^{39}$. Qu'une chambre de commerce et d'industrie achète des pages de publicité dans un quotidien pour défendre le projet soumis à concertation, ou encore qu'elle négocie en coulisse avec le maître d'ouvrage, on jugera qu'est enfreint le principe d'une délibération que seule la procédure doit contenir.

De fait, les réticences des acteurs organisés de la société civile à l'égard des dispositifs institutionnalisés tiennent aux restrictions que ces derniers imposent à l'action collective. L'entrée en concertation suppose de façon plus ou moins explicite de renoncer à d'autres libertés politiques. Pendant l'échange, comme aux bords des débats, les manifestations sont jugées inopportunes. Plus encore, l'offre de participation peut être assortie de conditions explicites limitant les capacités d'action des individus et des organisations. En Autriche comme aux États-Unis, des concertations relatives aux aires aéroportuaires accueillent les populations riveraines et leurs organisations sous réserve qu'elles renoncent au préalable à tout recours en contentieux. C'est ainsi qu'à l'occasion d'un projet d'extension de l' aéroport autrichien de Vienne, des associations environnementalistes ont choisi de boycotter le forum de médiation institué par le pôle aéroportuaire dès lors qu'elles ne pouvaient se résigner à abandonner leur droit d'ester en justice ${ }^{40}$. Aussi, alors que les acteurs de la société civile organisée misent sur la fragmentation et la démultiplication des scènes d'échange et des logiques d'action, les espaces publics institutionnalisés cherchent à imposer des cadres procéduraux unitaires et exclusifs.

Les dispositifs participatifs institutionnalisés prétendent par ailleurs articuler délibération et participation active des citoyens, considérant qu'une large sollicitation favorise la constitution d'un public, sinon représentatif de la population idéalement visée, du moins

38. Jean L. Cohen et Andrew Arato, Civil Society and Political Theory, Cambridge, MIT Press, 1992.

39. En 2007, la décision ministérielle retenant le principe de la réalisation du contournement autoroutier de Bordeaux a ainsi été annulée par le tribunal administratif, annulation motivée par le fait qu'une décision du Comité Interministériel à l'Aménagement et au Développement du Territoire (CIADT) survenue avant l'issue du débat était de nature à entacher la procédure.

40. Frédéric Deroubaix et Sandrine Rui, « Arènes de débats et modes de régulation des conflits », dans Aéroport, environnement et territoires : quels indicateurs d'environnement pour quelles concertations avec les acteurs locaux et les riverains? Retour d'expériences étrangères en matière de développement durable des aires aéroportuaires des métropoles, dir. Guillaume Faburel, Rapport pour Aéroports de Paris, 2008, p. 77-103. 
suffisamment diversifié et pluraliste pour fonder de façon renouvelée une volonté générale. L'ambition affichée est de faire émerger des voix et des arguments non pris en compte par le système démocratique traditionnel. Cette ambition correctrice s'accompagne de techniques de sollicitation diverses : parfois, l'ouverture en grand des portes du débat paraît suffire pour associer des participants habituellement tenus à l'écart des scènes de délibération, parfois des mécanismes plus sophistiqués sont mobilisés pour construire un public (invitations ciblées, tirage au sort, constitution d'un panel par voie d'échantillonnage...). Par ailleurs, l'ambition corrective suppose d'accueillir et de traiter de façon équivalente les participants. Dans l'enceinte des débats publics, l'attention à l'égard des individus singuliers et des représentants institutionnels se veut indifférenciée. Ce principe donne lieu par exemple à une distribution par ordre alphabétique des tours de parole scrupuleusement minutés. Ainsi, il s'agit doublement de limiter l'asymétrie : d'une part, en ouvrant le dialogue aux acteurs ordinairement tenus à distance ; d'autre part, en traitant tout locuteur de façon égalitaire. L'espace public institutionnalisé entend donc participer à l'extension du domaine d'une communication libre de domination.

Ce principe est envisagé comme une condition nécessaire pour favoriser une délibération conçue comme un échange de points de vue et d'arguments ou encore « une communication visant à produire un changement d'opinion au moyen de propositions impersonnelles ou portant sur le long terme $»^{41}$. Les espaces publics institutionnalisés attendent des participants qu'ils ne se contentent pas d'importer des convictions élaborées à l'extérieur, mais qu'ils soient disposés à se faire un point de vue par la délibération. Dès lors, l'obsession des instigateurs de dispositifs participatifs est que les participants se comportent en hommes et femmes détachés de toute forme de dépendance. Ce régime normatif ne s'applique toutefois jamais de façon aussi pure : les participants singuliers savent aussi qu'ils peuvent être disqualifiés pour n'avoir aucun poids dans l'ordre de la représentation. Les formes émergentes de participation emprunteraient ainsi leur modèle de délibération à la démocratie d'assemblée ${ }^{42}$. Il est attendu que les participants puisent leur légitimité dans la représentation de leur communauté d'appartenance (sociale, ethnique, géographique...) ou encore de leur communauté d'intérêts, mais une fois entrés en concertation, ils ont à se comporter en citoyens libres et autonomes. Autrement dit : tout participant à un espace public institutionnalisé est identifié, recruté ou mobilisé selon son ancrage dans le monde social et politique, mais doit se placer sous un voile d'ignorance ${ }^{43}$ pour entrer dans un débat où chacun s'efforce de justifier ses prétentions au nom de l'intérêt général.

Ce régime normatif, par son ambiguité même, est potentiellement source de dissolution des identités collectives et de dévitalisation des antagonismes. Il explique les réticences des corps intermédiaires. Ces derniers ne dissocient pas de la sorte le travail de construction d'une identité collective du travail dialogique autour de l'enjeu des débats et de la défense des intérêts. Pour se constituer, les corps intermédiaires sont tenus de penser la délibération en lien avec la représentation. Ils sont ainsi attachés à la maîtrise des processus de construction des identités collectives, fonctionnant selon des règles propres de communication et de coopération : l'accumulation d'expertises, d'expériences et d'intérêts sert tout autant à donner une consistance au « Nous » mobilisé, qu'à travailler la question des faits et des significations sociales afin d'élaborer des revendications. La consistance d'un acteur social dépend de sa capacité à réaliser ce travail discursif et réflexif sans perdre de vue le sens de

41. Bernard Manin, Principes du gouvernement représentatif, Paris, Flammarion, 1996, p. 258.

42. Nicolas Rousselier, «Deux formes de représentation politique : le citoyen et l'individu », dans $L a$ Démocratie en France, t. 1. Idéologies, dir. Marc Sadoun, Paris, Gallimard, 2000, p. 247-331.

43. John Rawls, Théorie de la justice, Paris, Seuil, 1987. 
l'antagonisme qui fonde l'action collective. Les corps intermédiaires assument le fait que les arguments ne sont jamais neutres et impersonnels et qu'ils s'ancrent dans des rapports sociaux et des rapports de force qui structurent toujours les débats, qui en sont la matière même.

\section{Complémentarité critique et consistance démocratique}

Partageant les enjeux de l'offre publique de participation, les acteurs de la société civile, malgré les réticences, restent rarement longtemps à distance des scènes participatives. Non seulement ils ont appris à les envisager comme des ressources complémentaires sur le chemin du « jacobinisme amendé », mais ils ne se conforment jamais pleinement aux normes contraignantes que ces scènes imposent, agissant tout autant dans et aux bords des arènes participatives. D'ailleurs les faits indiquent que plus de vingt ans de déploiement de la démocratie participative n'ont pas conduit à la perte d'emprise des corps intermédiaires sur la décision publique ni à une baisse de la conflictualité ${ }^{44}$. Les dispositifs participatifs s'institutionnalisent sans transformer profondément le système institutionnel néo-corporatiste. Les logiques d'action des corps intermédiaires perdurent, qu'il s'agisse de leur capacité à constituer des espaces publics oppositionnels ou à investir les processus décisionnels et législatifs. Nous voudrions dès lors insister sur le fait que la contribution de la société civile organisée aux discussions ouvertes par l'offre publique de participation demeure essentielle : d'une part, pour donner une consistance politique aux discussions publiques, et d'autre part, parce que le déploiement des formes contemporaines de démocratie participative constitue une opportunité pour régénérer en retour les acteurs organisés de la société civile.

Ainsi, si la concurrence est réelle, la vitalité respective des espaces publics institutionnalisés et des corps intermédiaires dépend de leur articulation. Lorsque les espaces publics institutionnalisés sont désertés par les acteurs de la société civile organisée ou qu'ils les excluent, ils tournent à vide et s'épuisent. L'expression singulière des individus ne manque ni d'inventivité, de compassion, de perspective critique ou encore d'arguments fondés en raison. Mais, bien souvent, une fois réunis, les citoyens ordinaires ont tendance à neutraliser les divergences, à fonctionner au consensus, à s'entendre sur les plus petits dénominateurs communs : les faits retenus sont peu problématisés ; les significations sociales avancées conformistes ; et les divergences d'intérêt euphémisées. Nina Eliasoph a montré comment, dans le cas américain, un espace public de discussion pouvait générer des discours moins généreux, moins ouverts, et surtout moins politiques que les conversations privées et ce, à rebours des thèses participationnistes et délibératives. Ses subtiles observations et analyses révèlent ces processus d'évitement du politique ${ }^{45}:$ les citoyens s'entendent pour produire des conditions où s'exprimer ouvertement dans un esprit public et politique est perçu comme inconvenant, et où, paradoxalement, cette évacuation du politique est perçue par eux comme une condition pour agir ensemble dans le bien commun. De leurs côtés, les militants et les activistes rencontrés par Eliasoph ne dissocient pas de la sorte les exigences

44. Sur ce point, on se reportera aux données produites par Bruno Charlier pour le Groupe Manon sur les conflits environnementaux. Leur nombre n'a en effet cessé de progresser après l'explosion des années 1989-1993 (327 conflits recensés pour la période 1994-1998, 337 pour la période 1999-2003), alors que, depuis cette période, les procédures de débat public et de concertation se sont multipliées et sont désormais systématiques en matière de projet de production et de transport. Groupe Manon, Horizon 2020 : Conflits d'usages dans les territoires : quel nouveau rôle pour l'État? Rapport pour le Commissariat général au Plan, Paris, La Documentation française, 2005 .

45. Nina Eliasoph, L'Évitement du politique : comment les Américains produisent l'apathie dans la vie quotidienne [1998], Paris, Economica, 2010. 
de la scène des discours publics et celles de la coulisse des conversations privées, jugeant qu'il n'y a pas « de moyen de séparer la politique du reste de l'existence », forgeant ainsi en continu un espace propice à la discussion.

Alors même qu'ils y contribuent, ce processus d'évitement du politique constitue une source de déception pour les citoyens ordinaires qui, passés l'enthousiasme des premiers moments de l'engagement participatif, s'interrogent sur l'utilité et le sens des dispositifs auxquels ils prennent part. Quand ils ne s'en détournent pas totalement. Dans une commune de Gironde, un dispositif sophistiqué de conseils de quartier n'a ainsi pas empêché le désenchantement de s'installer. Initialement, ces conseils devaient devenir des « corps intermédiaires », pour faire circuler les informations et jouer un rôle de transmission et de régulation entre l'espace social et l'espace politique, mais aussi des lieux de mise en commun d'expertises d'usage et d'expérience permettant à ces instances de proposer des projets et des actions pour améliorer le cadre de vie. Quatre conseils de quartier ont été institués, fondés sur un principe d'autonomie : ils étaient composés au moyen d'un tirage au sort au sein d'un vivier de volontaires, mécanisme assorti d'un principe de cooptation afin d'impliquer des citoyens aux profils généralement absents de ce type de dispositif (jeunes, handicapés, étrangers...), dotés d'une enveloppe budgétaire, et fonctionnaient hors la présence des élus. Le dispositif était complété par un comité d'éthique, instance de conseils et d'évaluation, mais aussi garant des principes fondateurs notamment celui de l'autonomie. La sophistication de la démarche, son esthétique démocratique et ses principes tiennent à un travail exemplaire en amont de l'institution proprement dite au sein d'une commission extra-municipale, s'efforçant de poser les bonnes questions afin d'éviter les pièges classiques de tout appel à la participation des habitants.

Mais, au terme de six ans de fonctionnement, l'essoufflement et la perplexité dominent. Les facteurs d'explication sont divers, mais, de façon centrale, la question du rôle et du positionnement de ce dispositif dans l'espace social et politique communal s'est posée de façon critique. Cette collection d'individus "volontaires" n'a rien d'autre à partager que cette envie de s'impliquer dans la vie de la commune. Elle ne peut compter ni sur la promotion d'un "programme commun", ni sur la défense d'une identité d'intérêts, toujours incertaine, ni sur la défense d'une cause fédératrice, à l'instar d'un collectif de mobilisation ou d'une association. "Le problème des conseils de quartier », constate un élu, « c'est que ce n'est pas une association : les gens ne se choisissent pas, ils n'ont pas un pôle d'intérêt unique, commun qu'ils partagent. » Au sein même de ces instances, la construction d'une parole commune suppose de traverser les épreuves des dynamiques de groupe, d'autant plus délicates ici que les membres peuvent ne pas partager les mêmes opinions et attitudes politiques, ne pas percevoir et définir les problèmes de façon similaire, ne pas partager la même vision de la gestion locale. Pour préserver un espace de collaboration et de discussion dont l'unité est fragile, le consensus est pris comme horizon, les participants recherchant l'accord sur les plus petits dénominateurs communs, logique contribuant à définir leur rôle de façon modeste et à limiter la créativité et l'innovation, et évacuant toute discussion politique.

Par ailleurs, l'embarras posé par l'autonomie et la légitimité litigieuse à représenter les habitants des quartiers explique les restrictions que les membres des conseils s'imposent, comme celles que leur impose, de façon plus ou moins explicite, la mairie. Car tant l'autonomie que la représentation sont des conditions équivoques dans le cas d'un dispositif de participation. Initiés par l'équipe municipale, qui fournit de surcroît une assistance technique, les conseils de quartier affrontent une ambiguïté jamais levée. Le «Soyez autonome » est une injonction bien paradoxale. Tout comme le doute persistant à l'égard de leur représentativité rappelle qu'une instance participative reste évaluée à l'aune des exigences de la démocratie de représentation. Les membres des conseils de quartier peinent 
à définir positivement leurs spécificités et à identifier une source alternative où puiser leur légitimité.

De fait, les participants rencontrés n'ont eu de cesse que de se montrer envieux à l'égard des acteurs organisés de la société civile qui produisent par eux-mêmes des espaces d'interpellation, de débat et d'action. Dans la commune, les composantes d'une société civile particulièrement active que sont les associations peuvent revendiquer une antériorité et une légitimité supérieure sur le terrain de l'autonomie et de la représentation, elles assument mieux leur rôle de partenaire critique à l'égard de la mairie, et n'envisagent pas les conseils de quartier comme une plus-value démocratique, estimant même que ces instances, loin d'être des relais, sont des écrans. Les membres des conseils perçoivent bien par ailleurs l'efficacité d'autres acteurs que sont les "collectifs", résultant de la mobilisation d'habitants autour d'un problème spécifique (ici des fuites d'eau, là un projet contesté de maison des services...). Quand l'offre publique de participation peine à mobiliser les habitants, les objets de contentieux constituent l'étincelle nécessaire à l'action collective et font sortir les gens de chez eux. Ces acteurs, éphémères, assument mieux que les conseils de quartier leurs fonctions critiques et contre-démocratiques. Dans le paysage civil communal, les acteurs en lutte savent toujours mieux se définir autour d'une identité d'intérêts que les membres des conseils de quartier qui se tiennent à distance du conflit. Or lorsque les espaces publics institutionnalisés refoulent ou nient toute dimension conflictuelle, ils deviennent au mieux des scènes d'information, de sensibilisation et de pédagogie civique qui, sans être inutiles, gagneraient à s'annoncer comme telles; au pire, ils ne relèvent que d'entreprises de pure légitimation dont le coût pourrait être épargné à la collectivité : même lorsqu'ils sont « ordinaires », les citoyens n'apprécient jamais de gaspiller leur temps dans des jeux de dupe. Ces scènes deviennent alors des lieux où se fabriquent le ressentiment et la défiance à l'égard de la vie démocratique qu'ils étaient pourtant censés réduire.

Mieux armés pour l'alerte et la problématisation, moins frileux à l'égard de la conflictualité, les acteurs de la société civile organisée sont des participants exigeants et leur contribution aux délibérations est souvent analysée comme un gage d'efficacité des dispositifs. Notamment quand ils ne se contentent pas d'instrumentaliser l'espace public institutionnalisé mais qu'ils en discutent et problématisent aussi la structuration et les règles du jeu ${ }^{46}$. Aussi, quand les acteurs de la société civile organisée optent pour le boycott ou l'indifférence, ils peuvent préserver leurs capacités d'action. Mais, outre qu'ils prennent le risque de la radicalisation et de l'isolement, ils passent surtout à côté d'une occasion de régénération, tant sur le plan des idées que sur celui de la représentation. Car en se confrontant aux individus singuliers sur les scènes participatives, il y a là l'occasion aussi d'un travail réflexif utile aux acteurs sociaux organisés. Ce travail ne s'observe pas aisément en situation de débat, tant les questions de légitimité, de représentativité et de responsabilité, particulièrement structurantes des discussions, donnent lieu à des joutes oratoires où la disqualification croisée est la règle. Toutefois l'exercice répété du débat et de la concertation, désormais étapes imposées des processus de décision, et la rencontre ainsi encouragée avec les participants ordinaires ne sont pas sans incidence sur les cadres

46. À partir de leurs études de cas brésiliennes respectives, Brian Wampler et Maureen M. Donaghy se rejoignent dans un même constat : là où la société civile est organisée et impliquée dans les dispositifs participatifs, ces derniers se caractérisent par une meilleure qualité procédurale et délibérative, tout à fait décisive pour que les discussions se traduisent en actions et mesures concrètes et profitables pour les populations. Voir Brian Wampler, «When Does Participatory Deepen the Quality of Democracy? Lessons from Brazil, Comparative Politics, 2008/1, p. 61-81 ; Maureen M. Donaghy, « Do Participatory Governance Institutions Matter ? Municipal Councils and Social Housing Programs », Comparative Politics, 2011/1, p. 83-102. 
de référence des corps intermédiaires. C'est le cas par exemple de l'intégration de la question sociale aux revendications des organisations environnementalistes. Ces dernières sont régulièrement tenues d'affronter en situation de débat public, l'expression d'individus, notamment des catégories sociales modestes, pour qui les exigences en matière de protection de la nature paraissent accentuer les difficultés rencontrées au quotidien ou contrecarrer des modes de vie et des usages prisés. Les temps de concertation peuvent se solder par des décisions et des mesures qui aggravent les inégalités sociales et environnementales ${ }^{47}$, mais il s'y travaille de fait des arguments et des justifications quant au caractère juste ou injuste de l'effort environnemental, qui nourrissent en retour les référentiels des acteurs environnementalistes.

Aussi, si les acteurs de la société civile organisée ont d'autant plus de capacité d'action et de capacité réflexive qu'ils participent d'un espace oppositionnel, si l'investissement continu dans l'administration consultative est un ressort nécessaire, l'engagement sur les scènes participatives est aussi source de renouvellement. Ils y trouvent des ressources d'action et de consolidation de leurs propres arènes discursives, mais aussi des ressources d'élargissement de leur surface de représentation, notamment parce que l'offre de participation institutionnalisée précipite la rencontre entre publics forts et publics faibles. C'est d'ailleurs en assumant la tension entre les différentes scènes de dialogue et d'action, et en garantissant la circulation des enjeux de discussion des unes aux autres, qu'ils contribuent à la consistance sociale et politique de la démocratie.

Dans un pays comme la France où la démocratie participative se développe tout en demeurant subalterne par rapport à la démocratie de représentation, on peut comprendre que l'engagement continu dans les mécanismes néo-corporatistes paraisse plus payant aux corps intermédiaires. Néanmoins, l'adhésion distanciée demeure bien plus souvent la règle que le boycott. En dépit des normes contraignantes qu'ils prétendent imposer et dont ils tirent leur caractère toujours ambigu, les dispositifs participatifs reposent sur des principes de pluralisme qui les rendent perméables à l'action des acteurs de la société civile, qu'il s'agisse d'organisations de longue date stabilisées ou de mouvements sociaux émergents. Ces derniers font donc l'expérience répétée de dispositifs qui, non seulement n'épuisent jamais complètement leur capacité d'action ni leur capacité réflexive, mais peuvent aussi contribuer à les renforcer et les régénérer. À condition de se situer dans un entre-deux indispensable à la préservation de l'autonomie. Aussi, du point de vue de l'action comme de l'analyse, le déploiement des espaces publics institutionnalisés ne peut être pensé en occultant ce qui se joue à leurs bords, dès lors que c'est dans le va-et-vient entre la pluralité des scènes d'interactions que se travaille la tension entre les intentions des autorités publiques de gouverner la société et l'auto-organisation du social.

Surtout, l'investissement des arènes participatives leur permet tout autant d'exercer une influence sur la formation institutionnalisée de l'opinion et de la volonté que d'actualiser les interprétations publiques qu'ils donnent aux expériences et aux intérêts sociaux. Pour les acteurs de la société civile organisée, l'enjeu est bien de rester en prise avec un corps social dont les composantes individuelles s'affranchissent toujours davantage des organisations, des registres et des répertoires traditionnels d'action collective, dans un rapport de distanciation et de défiance désormais très général à l'égard de toute forme d'intermédiaire.

UNIVERSITÉ DE BORDEAUX - CENTRE ÉMILE-DURKHEIM (UMR 5116)

47. Valérie Deldrève et Jacqueline Candau, « Produire des inégalités environnementales justes ? », Sociologie, 2014/3, p. 255-269.

हु $n^{\circ} 1,2016$ 